\title{
LA MELANCOLIA ADUSTA EN SAN ALBERTO MAGNO
}

\section{Fernando Pagés Larraya Gerardo Pagés}

Alberto de Bolstädt (1206-1280) (1), llamado Magnus por su saber oceánico, fue admirado aun por su crítico contemporáneo más severo, el franciscano de la escuela de Oxford Robert Bacon (1214-1294), creador con él de la scientia experimentalis del medioevo.

Alberto Magno asimiló el saber universal del siglo XIII, pudiendo tratar por ello los temas más diversos, desde los herméticos misterios de la teología hasta las virtudes de las plantas, los animales y las piedras.

El «tinte aristotélico de San Alberto Magṇo» (2) confundió a algunos tratadistas que llegaron a considerarlo tan sólo un singular compilator y no es aventurado afirmar que ha sido casi contemporáneamente cuando los especialistas en el saber medieval hari visto en él un auctor de singular genialidad.

El abertinismo pantagruélico consagrado por Etienne Gilson ha despertado una noble curiosidad acerca de la vida del Santo $(3,4,5)$ dado que su obra supera por su extensión y profundidad las más extraordinarias posibilidades humanas.

Una fuente excelente para el desciframiento del poderoso enigma de su existencia laboriosa y creativa es, sin duda, el ensayo de James A. Seisheipel; de la Orden de los Predicadores, que ha ordenado informaciones biográficas dispersas en una síntesis diacrónica, dentro de la obra compilada 
por Manfred Entrich, O. P., titulada adecuadamente Albertus Magnus, sein Leben und seinen Bedeutung.

Existen diversas percepciones de nuestro auctor, que surgen del análisis inacabado de su obra de magnitud inigualada.

Dante, en Paradiso X, 97, lo ubica en el cielo del Sol, hermanado a Santo Tomás de Aquino:

"Questi, che m'è a destra più vicino frate e maestro fummi, ed esso Alberto è di Cologna, ed io Thomas d'Aquino...».

Ambos son iluminados por la Quinta Luz (Paradiso, X, 109):

"La Quinta Luce, ch’è tra noi piú bella, Spira di tale amor, che tutto'l mondo la ggiù ne gola di saper novella».

La presencia de Alberto Magno en el Paradiso de Dante Alighieri recoge una tradición muy difundida de su persona, la de heraldo de Tomás de Aquino, y piedra angular del pórtico de la escolástica.

Desde sus tiempos primeros 'se dibujó, sin embargo, una imagen esotérica del Santo, como mago y alquimista, autor de grimorios y profecías innumerables $(6,7,8,9)$.

Su omnisciencia hizo también que en el curso de los tiempos su obra apareciera fragmentariamente en los paradigmas más diversos de la historia del pensamiento $(10,11,12,13)$.

A nadie, sin embargo, escapó su innovación trascendental consistente en la separación de la teología de la filosofía, haciendo de ésta una ciencia sensu stricto.

Podríamos afirmar, empero, que:Alberto Magno es todavía un pensador desconocido en múltiples aspectos, como lo evidencia la renovación de su estudio luego de la prolija restauración de sus Opera Omnia que lleva a cabo actualmente la Orden de Predicadores, a través del concilium de sabios albertinianos realizado por el Monasterium Westfalorum in Aedibus Aschendorf.

El tratamiento moderno de sus textos ha hecho de ellos un venero de sabiduría y originalidad:

Nuestro episcopus ratisbonensis, que vivió bajo el cielo de Ptolomeo e iluminó.su universo con las luces de Dionisio el Areopagita, aplicó la scientia experimentalis, provocando una renovación creativa del saber, de lo que 
será un testimonio su tratamiento de la melancolía, una verdadera Aufhebung (elevación y síntesis) a partir de los Problemata de Aristóteles y la estequiología de Galeno (14):

Como introducción al tema de nuestro ensayo, se impone en la gnoseología albertiana una breve discriminación de tres nociones, que no deben ser confundidas, sobre las que imperó, a veces, una insalvable confusión en los medievalistas; son ellas las de tristitia, acedia y melancolía.

Ioannes Cassián trató en su obra De Institutis Ceonobiorum et de octo principalium vitiorum remediis (Siglo V ) (15), «De Spiritu Tristitiae» de la siguiente manera:

Est etiam aliud detestabilius tristitiae genus, quod non correctionem uitae nec emendationem uitiorum, sed perniciosissimam desperationem animae inicit delinquenti: quod nec Cain fecit post fratricidium paenitere nec Iudam post proditionem ad satisfactionis remedia festinare, sed ad suspendium laquei sua desperatione pertraxit.

«Hay otro género de tristeza más detestable, que no impulsa al culpable a rectificar su vida o corregir sus vicios, sino que lo lleva a una desesperación mortal: él ha impedido a Caín arrepentirse después del fraticidio, y que Judas, después de su traición se apresurara a reparar su falta, sino que lo llevó a que se ahorcara por desesperación».

(«De Spiritu Tristitiae», Liber Nonvs, 9)

De «Spiritu Acediæ» (16) fue objeto de otro importante tratado de Ioannes Cassianus:

Sextum nobis certamen est, quod Graeci $\alpha \kappa \eta \delta \mathrm{i} \alpha$. uocant, quam nos taedium siue anxietatem cordis possumus nuncupare. Adfinis haec tristitiae ac solitariis magis experta et in heremo commorantibus infestior hostis ac frequens, maxime circa horam sextam moriachum inquietans, ut quaedam febris ingruens tempore praestituto ardentissimos aestus accessionum suarum solitis ac statutis horis animae inferens aegrotanti. Denique nonnulii senum hunc esse pronuntian meridianum daemonem, qui in psalmo nongesimo nuncupatur.

«En sexto lugar vamos a combatir lo que los griegos llaman acedia y que nosostros podemos denominar tedio y ansiedad del corazón. Vecino a la tristeza, este adversario prueba sobre todo a los solitariọs, y ataca más frecuente y duramente a aquellos que moran en el desierto. Es sobre todo alrededor de la hora sexta cuando perturba al monje, excitándolo a horas 


\begin{abstract}
fijas, como una fiebre que retorna periódicamente a su alma enferma por los ardores violentos qué provoca. En fin, algunos ancianos declaran que es el demonio del mediodía del que habla el Salmo XC»
\end{abstract}

(«De Spiritu Acediae». Liber Decimus, 1)

La fuente albertiana de la melancolía se halla constituida por los Problemata de Aristóteles $(17,18,19)$ en los que es objeto de un trascendente discurso: Problemata, XXX, 1.

Es interesante destacar que Aristóteles planteó a través de ellos un poderoso interrogante, quie a veces se difundió como si se tratara tan sólo de un dictum, tal como figuró en la obra de Cicerón (20).

El texto inicial de Problemata XXX, 1, dice así:

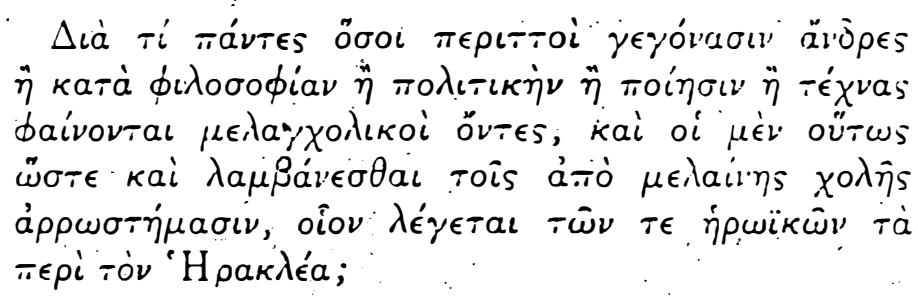

" ¿A qué se debe que todos ellos que han llegado a ser notables en filosofía, política, poesía o artes, parecen ser melancólicos, y algunós hasta el punto de que se apoderan de ellos las dolencias que provienen de la bilis negra, como lo narran las historias heróicas acerca de Heracles?

(Aristóteles, Problemata XXX, Cap. 1, 953 a)

Un análisis minucioso de la obra de San Alberto Magno nos ha permitido advertir sú original respuesta al problema pseudoaristotélico de la melancolía formulado unos quinientos años antes de Cristo.

Para su exégesis hemos elegido tres textos raigales que nutren el frondoso árbol de nociones y comentarios acerca de la melancolía albertina, que trascienden, por cierto, la respetuosa brevedad de nuestro ensayo.

En el Lib. I, De Somno et Vigilia, Trat. II, Caput V, De his qui faciunt in somno opera vigilantium, Vol. IX, de Opera Omnia de la edición de August Borgnet, titulado Parvorum Naturalium Pars Prima, afirma Alberto Magno haber conocido el dictum Aristotélico a través de excerpta: «... dictum est in libro de Problematibus ab Aristotele, qui liber non ad me pervenit, licet viderim quaedam excerpta in ipso», p. 145. 
Por otra parte, en la extensa disgresión referida a la diversidad de humores naturales y no naturales, que aparece en el Vol. XI de sus Opera Omnia-Animalium Lib. XXV I (Pars prior, I-XIII), Caput III- revela un conocimiento profundo de la obra de Constantino Africano, al que cita explícitamente: «Melacholia autem quae cholera nigra sive nigra kilis vocatur, est ultimus humorum naturalium, sicut testatur Constantinus" (pág. 238).

En este discurso expresa que antes de determinar la naturaleza y generación de los miembros o elementos que se constituyen en los cuerpos animados, como la carne, la sangre, la leche y el esperma, es menester que derivemos a la doctrina general de los seres húmedos.

El humor del cuerpo animal es un fluido líquido en el cual se conviérte el alimento nutritivo por medio de la segunda digestión. Esta es llamada así ya que es realizada en el hígado o en el órgano que lo reemplaza.

La primera digestión tiene lugar en el estómago. El resultado de ambas digestiones es el humor, que puede ser bueno o malo.

El humor bueno se divide y subdivide. El primer grupo incluye la sangre, la pituita, la bilis roja y la bilis negra.

Trata extensamente de Alberto Magno los diversos entes que instituye en esta división y subdivisión, analizando con ejemplificadora claridad y conocimiento profundo los tipos de sangre, de la pituita (phlegma) y de la bilis.

Al hablar de la bilis citrina - p. 237-, se refiere a la melancolía llamada no natural o adusta (quemada, abrasada). Esa adustión se realiza en la misma bilis.

El comentario de la adustión de la bilis deja vislumbrar un conocimiento perfecto de la estequiología de Galeno, en un señalamiento claro y riguroso de los diversos tipos y efectos, así como los caracteres que revisten.

La melancolía, que también es llamada Cholera nigra o nigra bilis, es el último de los humores naturales de que trata Alberto en su disgresión, ahondando con ello conceptos de Constantino Africano (21).

Constantino Africano, miembro del famoso Collegium Hippocraticum, llevó a su máximo esplendor la Escuela Salernitana, y en lo referente a la melanćolía, trasmitió las tradiciones médicas helenísticas, bizantinas, árabes, latinas y hebreas.

El conocimiento de los tratados de la melancolía de Constantino Africano nos permite saber que Alberto Magno dispuso en su tiempo de la su- 
ma de las nociones existentes acerca de ese tema, fundando en ella su análisis singular de la melancolía de Aristóteles.

La lectura del siguiente texto, - Lib. III De Animalibus. Trac. II- nos permite apreciar la original concepción de Alberto Magno, de la melancolía aristotélica.

Hæc igitud melancholia naturalis fæx est sanguinis, sicut diximus, nec potest esse aliqua naturalis melancholia quæ sit fæx et hypostasis aliorum humorum: quoniam a phlegmate per residentiam nihil separatur propter sui viscositatem, sicut neque ab oleo. A cholera naturali similiter per residentiam nihil de quo sit curandum, separari poterit propter frequentes motus ipsius, et subtilitatem, et paucitatem, terrestreitatis ipsius, et ejus levitatem: et ideo contingit melacholiam naturalem simplicem sanguinis. esse hypostasim. Licet autem ab aliis humoribus non separetur aliquid hypostaticum per modum residentiæ, segregatur tamen ex eis aliquid terrestre quod est in ipsis, aut per calorem adurentem, aut per figus exprimens. Sed hoc modo generatur melancholia innaturalis: et ideo innaturalis melancholia non est fæx et hypostasis sanguinis. 'Et-licent etiam cholera quam supra vocavimus adustam, etiam sit adustio cinerea, tamen sicut supra diximus, in eadem cholera manet subtile acutum et humidum: hoc autem non manet in melancholia cinerea adusta: et in hoc est differentia adustionis cinereæ. in cholera rubea, et in melancholia innaturali.

Hæc igitur innaturalis melancholia est quadruplex. Una quidem quæ a cholera rubea per adustionem separatur, in qua non manet subtile humidum, quia evolavit, sed sola manet cinereitas quæ melancholia vocatur. Alia autem separatur per adustionem a phlegmate: et hoc dupliciter, sicut duplex in genere est substantia phlegmatis. Quædam enim separatur a subtili phlegmate per adustionem cineream, et hæec est salsa parum tendens ad ponticitatem, et non est multum mala. Quædam autem per adustionem separatur a phlegmate grosso, et hæc aliquando est acris, et aliquando pontica, aut forte commixti saporis: ńeque etiam ista est multum nociva. Tertia autem separatur per adustionem a sanguine quæ est saporis pontici parum vergentis ad dulcedinem, et rion est valde nociva etiam ista: Quarta autem separatur a melancholia: et hoc contingit duobus modis. Aliquando enim per adustionem cineream separatur a melancholia naturali subtili, et hæc est nigra acris et acetosa bulliens, quando cadit super terram per egestionem, cujus gustum fugiunt muscæ, et alia animalia humidum stercoris gustantia, et hæc pernecabilis est et excoriativa, nisi cito occurratur ei in principio, et habet insuper hanc malitiam, quod non de facili recipit medicinam. Aliquando autem per adustionem separatur a melancholia grossa, et hæc est minoris acredinis, paucam habens ponticitatem: tardius autem nocet quam ea de qua 
jam ante diximus: sed habet hanc malitiam, quod etiam tardius quam illa recipit medicinam. Isti igitur sunt modi melahcholiæ: et ea quidem quæ est naturalis; abundat in multis animalibus, ita quod totus sanguis eorum est melancholicus, sicut bubali et viri et boves qui apud nos vuesent vocantur: et similiter est in aliis animalibus.

In hominibus autem etiam diversimode participatur humor iste: quoniam si non sit multum adusta, sed sit adustio aliqua cum melancholia naturali quæ alteret ipsam et' non incineret; ita quod fumare incipiat, tunc melancholia illa erit habens multos et stabilitos et confirmatos spiritus: quia calidum ejus bene miovet, et ejus humidum cum hypostasi terrestri non incinerata optime movetur: propter quod tales habent stabilitos conceptus et ordinatissimos effectus, et efficiuntur studiosi et virtutum optimorum. El ideo dicit Aristoteles in libro de Problematibus, quod omnes majores Philosophi sicut Anaxagoras et Thales Milesius: et - omnes illi qui virtutibus præcellebant heroicis, sicut Hector, et Eneas, et Priamus; et alii, erant de tali melancholia. Dicunt enim, quod talis melancholia habet naturam vini rubei, quod fumosum est, et confirmatorum et stabilium spirituum generativum.

Hæc igitur de melancholia a nobis dicta sunt:

«Por lo tanto, esta melancolía es sedimento natural de la sangre, como hemos dicho, y no puede haber melancolía natural alguna que sea sedimento "sustancia" de otros humores, puesto que nada se separa del humor viscoso por ásiento o causa de su misma viscosidad, así como tampoco del aceite. Similarmente, nada valedero podrá separarse por decantación de la bilis natural a causa de sus frecuentes movimientos, su sutileza, su escasa densidad, su levedäd, y por lo tanto, acaece que la melancolía natural es de simple naturaleza sanguínea. Áunque algo sustancial no se separe de los otros humores por decantación, con todo se separa de ellos algo denso que está en ellos, ya por calor ardiente, ya por frío que comprime. Pero de este modo se engendra la melancolía no natural:y de allí que esa melancolía no natural no es sedimento y sustancia de la sangre. Y aunque la bilis sea esa que antes llamamos quemada, abrasada de cenizas, con todo, según dijimos, en esa misma bilis permanece algo finamente sutil y húmedo, esto, empero, no queda en la melancolía ardida cenicienta: y en esto radica la diferencia de la adustión cenicienta en la bilis rubina o roja y en la melancolía no natural.

Por lo tanto, esta melancolía no natural es cuádruple. Una, pues, que de la bilis roja se separa por adustión, en la que no permanece lo húmedo sutil, porque se ha evaporado, y'sólo resta lo ceniciento que es llamado melancolía. Otra se separa por adustión del humor viscoso y esto de dos maneras, como es doble, en general, la sustancia del humor viscoso. 
Pues alguna se separa del humor viscoso sutil por adustión cenicienta, y ésta es algo salada; tendiendo al sabor del mar, y no es muy mala.

La tercera empero, se separa de la sangre por adustión y tiene sabor a mar, algo tendiente a la dulzura; tampoco ésta es muy nociva.

La cuarta se separa de la melancolía, y esto ocurre de dos maneras. Alguna vez por adustión cenicienta se separa de la melancolía natural sutil, y esta es negra, acre y avinagrada al bullir, y si cae sobre la tierra derramada, las moscas huyen de su sabor, y lo mismo otros animales que gustan de la humedad del estiércol, y ella es mortal y provoca excoriaciones, a menos que se acuda rápido y desde el comienzo, y además tiene la dificultad de no ceder fácilmente a la medicina. A veces se la separa por adustión de la melancolía gruesa, y ésta es menos acre, con poco sabor a mar. Daña más lentamente que aquella de la cual ya hemos hablando, pero presenta la dificultad de que también cede más lentamente a los medicamentos. Estas son, pues, las clases de la melancolía, y aquélla que es natural abunda en muchos animales, al punto de que toda la sangre de ellos es melancólica, como los búfalos y los machos y los toros que entre nosotros son.llamados vuesent, e igualmente suçede en otros animales.

En los hombres también este humor forma parte de diversas maneras: porque si no hay gran abrasamiento, sino que se produce una cierta adustión con la melancolía natural que la altera sin incinerarla, de modo que comience a echar humo, entonces tendremos una melancolía con muchas fuerzas firmes y consolidadas, pues su parte cálida se mueve bien y la húmeda lo hace muy bien con la sustancia terrestre no incinerada, por lo cual produce efectos y frutos tan firmes y adecuados, siendo origen de las virtudes de los mejores. Por ello, dice Aristóteles en el libro de Problematibus que todos los mayores filósofos, como Anaxágoras y Tales de Mileto, y todos aquellos que sobresalían por sus virtudes heróicas, como Héctor y Eneas, y Príamo y otros, eran de tal melancolía. Dicen, pues, que tal melancolía tiene la naturaleza del vino rojo (o tinto) que despide humo (es espirituoso) y que engendra espíritus afirmados y estables. lía».

Estas cosas, pues, han sido dichas por nosotros acerca de la melanco-

El señalamiento del spiritus generativus de la adustión de la nigra bilis, que infunde el genio a los melancólicos de Aristóteles, es sin duda una creación de absoluta originalidad de San Alberto, llevada a cabo mediante una síntesis que aúna en una nueva estructura, saberes a veces contradictorios. 
Es esta una iluminación que permite dar una respuesta cabal al interrogante de Problemata XXX, 1 que aún nos conmueve por su poderoso enigma.

\section{NOTAS}

(1) Gilson, E. (1944): La Philosophie au Moyen Âge des Origines Patristiques à la Fin duXIVe. Siècle. París, Payot (Deuxième édition...). Chap, VIII. «La Philosophie au XIII e. Siècle. D'Albert le Grand a Dietrich de Freiberg», pp. 503-525.

(2) Schipperges, H. (1972): "La medicina en la Edad Media». En: P. Laín Entralgo, Historia Universal de la Medicina. Barcelona, Salvat Editores, S. A. p. 240.

(3) Von LoE, P. (1900-1902): «De vita et scriptis B. Alberti Magni». Analecta Bollandiana, XIX, pp. 272-284; XX, pp. 273-316;XXI, pp. 361-371.

(4) De Reaymaker, L. (1933): «Albert le Grand Philosophe. Les lignes fondamentales de son système métaphysique». Revue Neoescolastique, XXXV, pp. 5-36.

(5) Weisheipel, J. A., O.P. (1982): «Albert der Grosse, Leben und Werke». En: Manfred Entrich, OP (Hrsg).), Albertus Magnus, sein Leben und seine Bedeutung, Graz, Wien, Köln, Verlag Styria, pp. 9-60.

(6) Alberto Magno (1978): Alberti Magni Liber Aggregationis, seu secretorum de virtutibus herbarum et animalium quorundam ejusdem de mirabilibus mundi seu de secretis naturae et alia opuscula. Bononiae, per Joannem Schriber de Annuntiata de Augusta, 1.

(7) Alberto Magno (1806): Le Grande et Véritable Science Cabalistique, ou la Sorcellerie Dévoilée, Contenant: $1 .^{\circ}$ Le Grand Albert et ses Merveilleux Secrets; $2 .^{\circ}$ Les Secrets Mystiques de la Magie Naturelle du Petit Albert; $3 .^{\circ}$ Le Dragon Rouge, París, Le Bailly.

(8) Alberto Magno (1700): Admirables Secrets de Grand Albert, Contenant un Traité sur La Vertu des Herbes, des Pierres Précieuses et des Animaux. París, chez tous les marchands de nouveautés (Lagny, impr. de Giroux et Vialat, 1850).

(9) Alberto Magno (1722): Secrets merveilleux de la magie naturelle et cabalistique du Petit Albert, traduits exactement sur l'original latin qui a pour titre: Alberti Parvi Lucci Libellus de Mirabilis Naturae Arcanis... Cologne, aux dépens de la Compagnie, 1722.

(10) Beati Alberti Magni, Ratisbonensis Episcopi Ordinis Praedicatorum Opera Quae Hactenvs Haberi. Potvernvt, SVB Rnis P. P. F. F. Thoma Tvrco, Nicolai Rodulpho, Ioan, Baptista De Marinis... Tomus Primus, Lugduni, Sumptibus Claudii Rigaud, Frat, Hironymi de La Garde. Ioan. Ant Hugvetan Filis, M. DC. LI, Catalogus:Operum Beati Alberti Magni XXI...

(11) Beati Alberti Magni..., Opera Omnia, ex Editione Lugdunensi Religiose Castigata, et Pro Auctoritatibus ad Fidem Bulgatae Versinis Accuratiorumque Patrologiae Textuum Revocata, Auctaque B. Alberti Vita ac Bibliographia Suorum Operum a PP. Quétif et Echard. Exaratis, Etiam Revisa et Locupletata, Cura ac Labore Augusti Borgnet, "Parvorum Naturalium» Pars Prima, Lib. I, De Somno et Vigilia, Tract. II, Caput V, p. 145. Caput VIII, pp. 205-206, Vol. Nonum; Animalium, Lib. XXVI, Tract. II, Caput III, pp. 233-240, Vol. Undecimum; Ethicorum Lib X; Liber VII Ethicorum, Trat. II, Caput V, pp. 508-512; Vol. Septimum... Parisiis, Apud Ludovicum Vivés, Bibliopolam Editorem, MDCCCXCI. 
(12) Sancti Doctoris Ecclesiae Alberti Magni, Opera Omnia, De XV: Problematibus. Edidit Bernhardus Geyer. VII. Quod Anima, quae est forma hominis, secundum quod homo, corrumpitur corrupto corpore $« \ldots$ si autem isti antique erroris novi vellent esse discipuli, qui a Platone derivatus est, et dicere velint, quod vegetabilis et sensibilis et rationalis tres sint substantiae, quae sunt in homine, facile hoc eliditur per Priman Philosophiam", Tomus XVII, Pars, I, p. 40, Monasteri Westfalorum in Aedibus Aschendorff, 1975.

(13) Fauser, W., S. J. (1982): Die Werke des Albertus Magnus in ihrer Handschriftlichen überlieferung. Teil I, Die Echten Werke Codices Manuscripti Operum Alberti Magni Opera Omnia. Tomus Subsidiarius I, Pars. I, Monasterii Westfalorum in Aedibus Aschendorff.

(14) Claudit Galeni: Opera Omnia. Editionem cvravit C. G. Kühn. Tomus I, pág. 522; T. IV, p. 509, V. 666-609 (Spiritus in ventriculis cerebri); VII, pág. 844; XV; 802; XVII, 244; XVII, B. $248,385,654$; XVIII, A. $142 \ldots$

(15) CASSIEN, J. (1965): Institutions Cénobitiques. Liber Nonus. «De Spiritu Tristitiae». Texte Latin Revu. Introduction et Notes par J. C. Guy, S. J. París, Editions du Cerf, pp. 368381.

(16) Pagés Larraya, F. (1980): «De Spiritu Acediae». Psychologica. Revista Argentina de Psicología Realista, n. ${ }^{\circ}$ 4, En./Jun. pp. 137-238.

(17) ARISTOTELES (1927): The Works of Aristotle. Translated into English under the Editorship pf. W. D. Ross. Vol. VII. Problemata, by E. S. Foster, Book XXX, 1953 a... Oxford, At. the Clarendon Press.

(18) Foster, E. S. (1929): «The pseudo-aristotelian problems: his nature and composition». Classical Quarterly, XXII, 1929, pp. 163 ss.

(19) La CROCE, E. y Pages LarRaya, F. (1979): «Un tratado aristotélico sobre la melancolía». Psychologica, n. ${ }^{\circ}$ 3, jul/dic. 1979, pp. 79-94.

(20) MARCII TUlLiI Ciceronis'(1865): Tusculanae disputationies." Libri V cum commentariis. Joännis Davisii. Halis Saxonum Sumtibus; C. A. Kummelii, 1805. I. 80, «... Aristóteles quidem omnes ingeniosus melancholicos esse».

(21) Pages Larraya, F. (en prensa): Introducción a los textos de la melancolía: Constantini Africani "De Melancholia". Buenos Aires, Alianza Editorial. 